\title{
Citation and referencing guidelines
}

\author{
James Dibley (iD) sacj.production.editor@gmail.com, Philip Machanick (iD sacj.editor@gmail. \\ com \\ Department of Computer Science, Rhodes University, South Africa
}

\section{INTRODUCTION}

This short article provides some guidelines and worked examples of reliable citation and referencing practice for contributing authors and reviewers to/for the South African Computer Journal. This is motivated by several observations:

1. that citation data obtained through search engines and reference managers are often unreviewed, misformatted, or incomplete;

2. while $S A C J$ is prepared for publication using the $\mathrm{BT}_{\mathrm{E}} \mathrm{X}$ toolchain, a great number of accepted contributions to the journal are prepared using other software, and in these cases, $S A C J$ production staff must manually transfer the citation data;

3. better guidelines for contributors and reviewers can support more rigorous review, resulting in a faster and more effective publication pipeline for SACJ overall.

As such, this article aims to provide enough detail:

- to prepare an article for submission, and

- to establish that a submitted article is ready to be accepted for publication.

\subsection{Background}

For historical and interdisciplinary reasons, SACJ published articles employ a citation and referencing style that closely tracks the American Psychological Association style guide, 6th edition. We are greatly assisted in this by Philip Kime's biblatex-apa6 package ${ }^{1}$.

Submissions to SACJ are not required to use the APA 6th citation style, and we recommend that contributors do not attempt to do so.

However, SACJ does recommend that supplied reference sets include all of the data required by the APA 6th referencing style. Section 3 details this.

Dibley, J. and Machanick, P. (2021). Citation and referencing guidelines [Communication]. South African Computer Journal 33(2), 72-78. https://doi.org/10.18489/sacj.v33i2.1048

Copyright (C) the author(s); published under a Creative Commons NonCommercial 4.0 License (CC BY-NC 4.0).

SACJ is a publication of the South African Institute of Computer Scientists and Information Technologists. ISSN 1015-7999 (print) ISSN 2313-7835 (online).

${ }^{1}$ https://www.ctan.org/pkg/biblatex-apa6 


\section{SUBMISSION GUIDELINES}

\subsection{Citation style}

Authors using LaTeX, Microsoft Word or alternative word processing software should prepare their submissions using an IEEE-style numeric citation style, e.g.:

As Pade observes, "in introductory courses on quantum mechanics, the practice of formal skills often takes priority (this is subsumed under the slogan 'shut up and calculate')." [1, p. xvii]

[1] Pade, J. (2018). Quantum mechanics for pedestrians (2nd). Springer. https://doi.org/10.1007/9783-030-00464-4

This citation style enables the most direct workflow for reviewers to evaluate a submission, as well as the most direct workflow for the production editor to prepare a pre-publication proof.

\subsection{Referencing}

Please make best efforts to provide complete, up-to-date and correctly-styled reference entries at the point of submission. Submissions with inaccurate or incomplete referencing may result in significant delays to preparation of the final proof.

\subsubsection{Abbreviation}

Please do not abbreviate the titles of journals or conference proceedings. Where abbreviations have been used in downloaded reference entries, these should be corrected.

\subsubsection{Recommendations for contributors using Microsoft Word}

SACJ recommends that authors preparing submissions in Microsoft Word make use of one of several freely-available reference manager plugins ${ }^{2}$ that are able to export reference sets as BibTeX files. This simple step can significantly reduce time and risk of transcription error during the preparation of the final proofs.

The list of references at the end of this document may be used as a guide for manual preparation of reference entries, while the BibTeX code examples itemise the attributes required.

Numeric keys should be used for inline citations and references should use numeric keys ([1], [2-3], [4, 5, 6], etc.), rather than author names.

\subsubsection{Recommendations for contributors using LaTeX}

The SACJ production editor also cautions that BibTeX reference entries obtained through Google Scholar (e.g., through the 'cite search result' link) are captured from existing documents

\footnotetext{
${ }^{2}$ e.g. https://www.zotero.org/download/
} 
rather than automatically generated from article metadata, and as such may be poorly-styled, incomplete, or inaccurate.

Authors may consult the BibTeX entries provided in this document to check the style and information requirements.

\section{EXAMPLE REFERENCE ENTRIES}

\subsection{Article}

Aaaa and Bbbb (2047) is provided by the following BibTeX code:

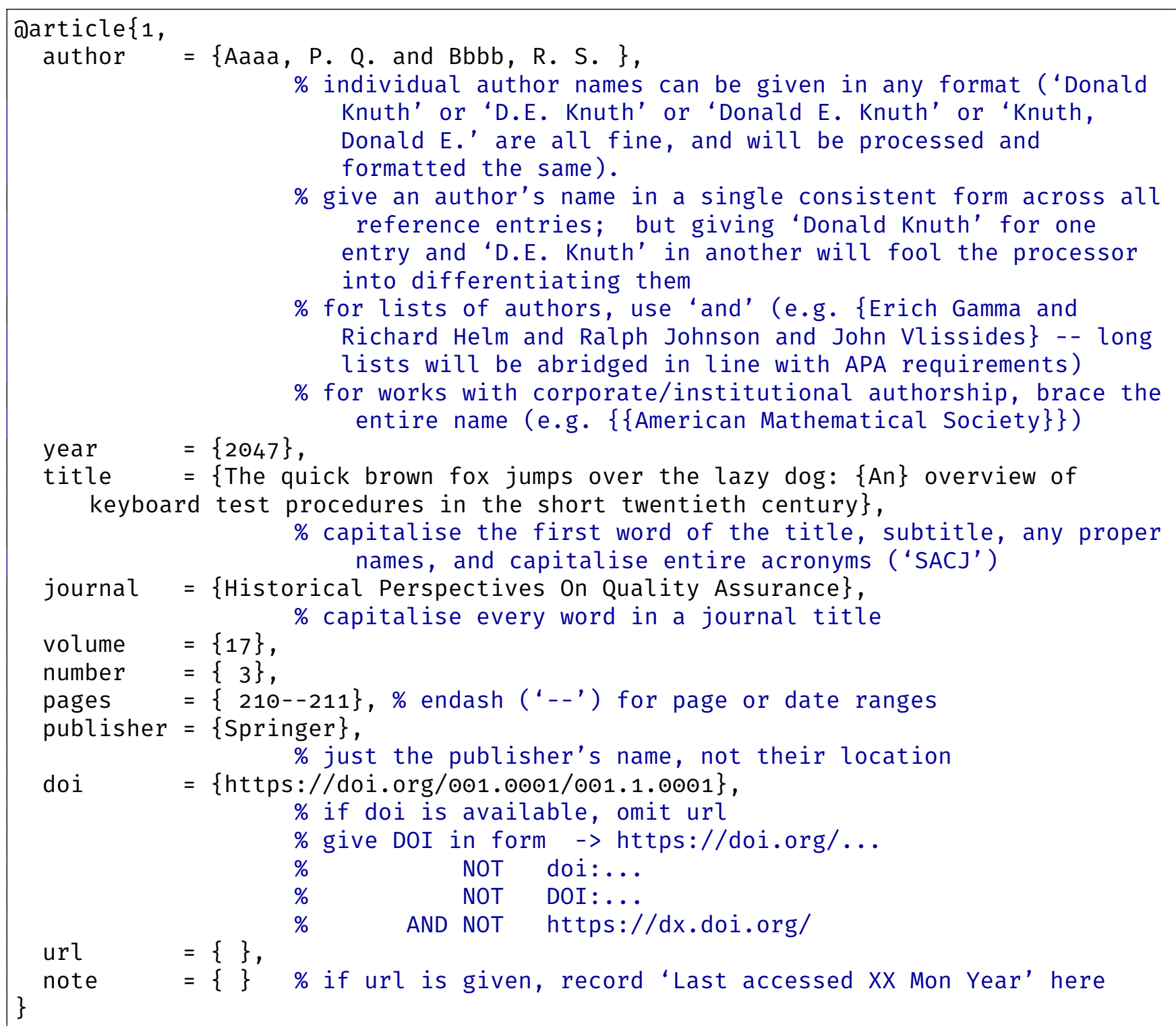




\subsection{Conference paper}

Cccc (2017) is provided by the following BibTeX code:

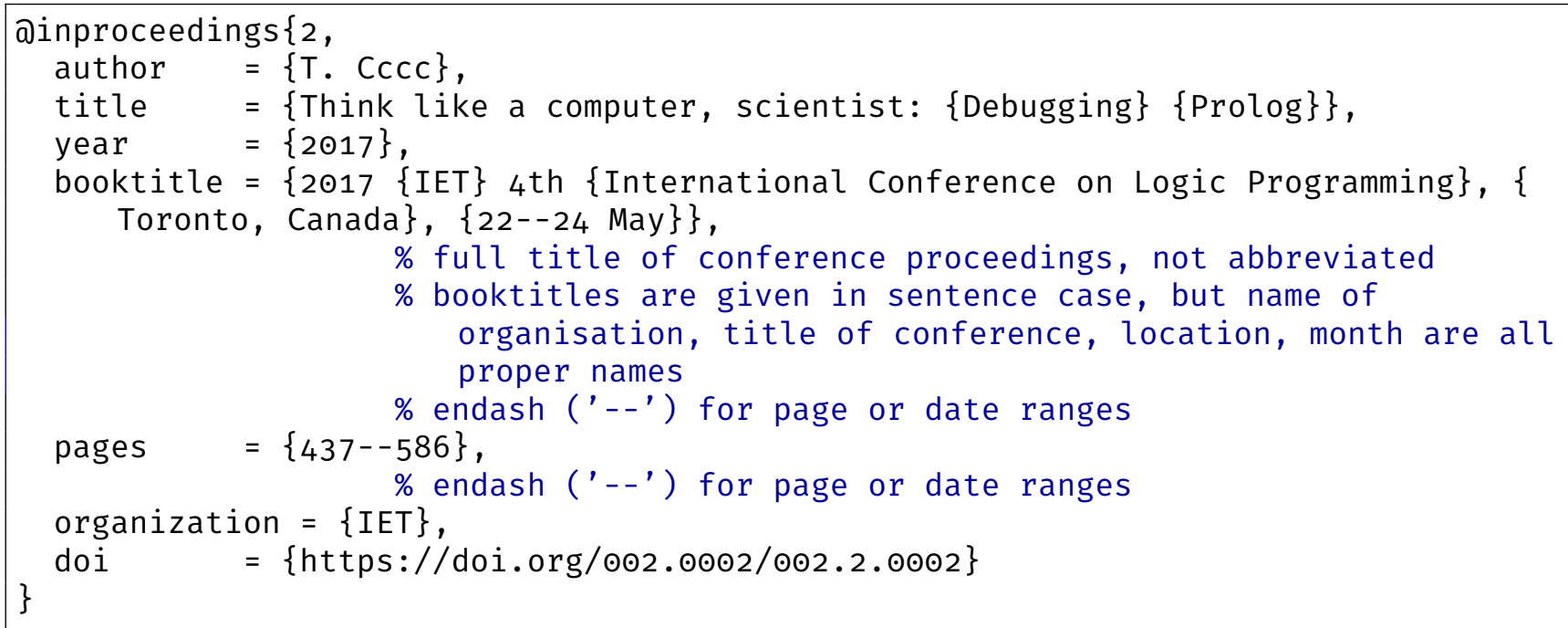

\subsection{Book}

Dddd (2008) is provided by the following BibTeX code:

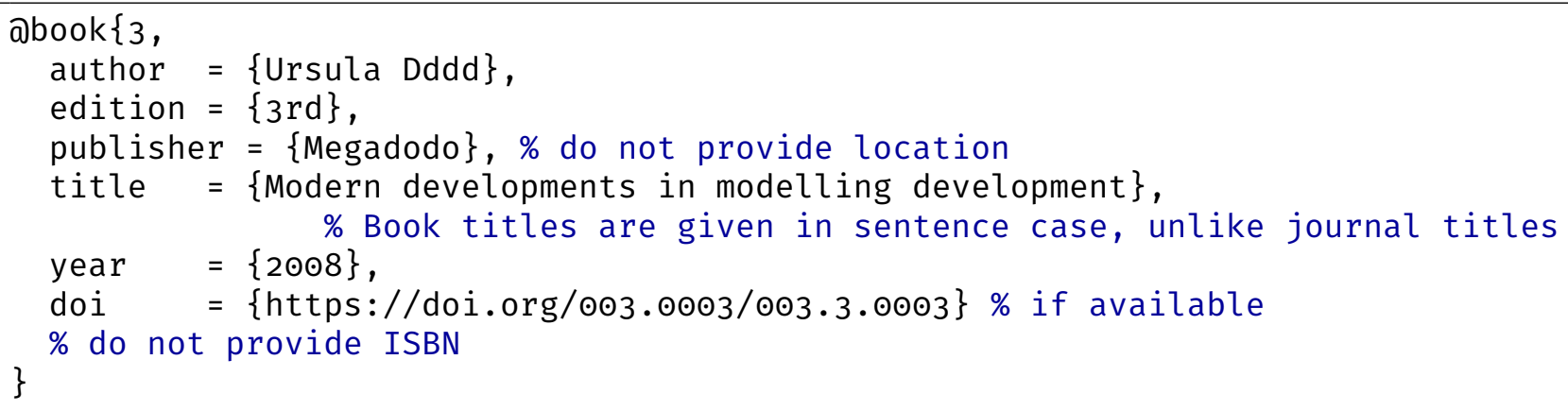




\subsection{Book chapter}

Eeee (1998) is provided by the following BibTeX code:

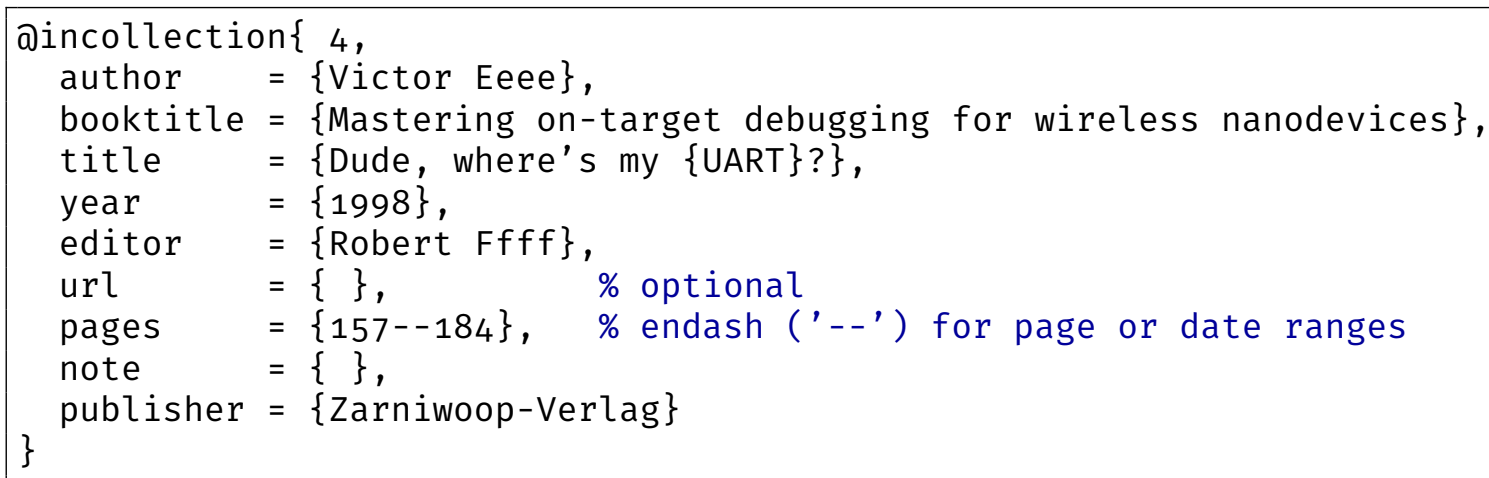

\subsection{Theses}

Gggg (1888) is provided by the following BibTeX code:

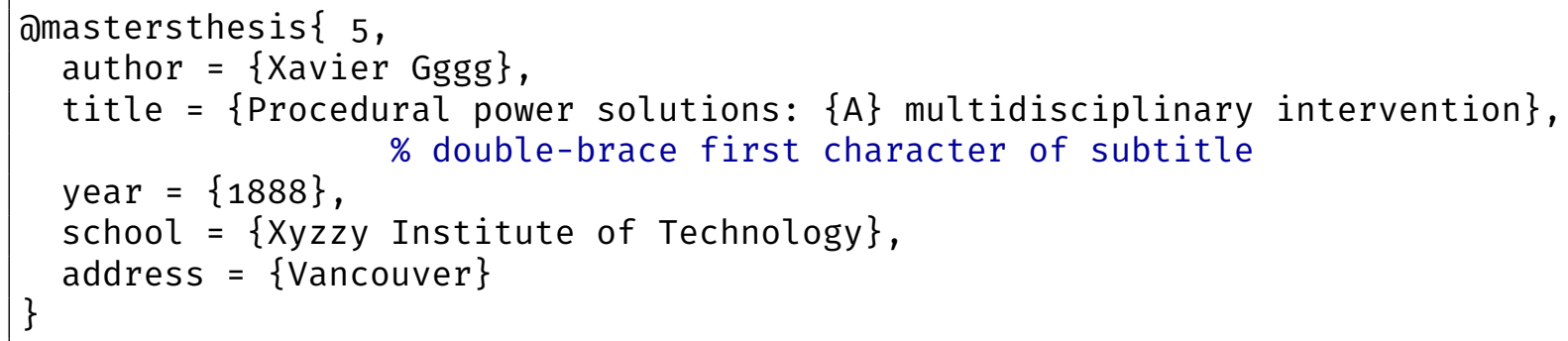

Hhhh (1999) is provided by the following BibTeX code:

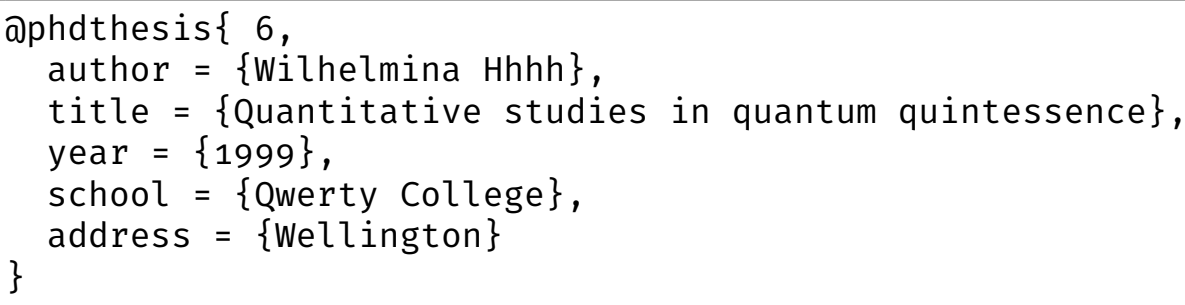




\subsection{Technical report}

Iiii (2000) is provided by the following BibTeX code:

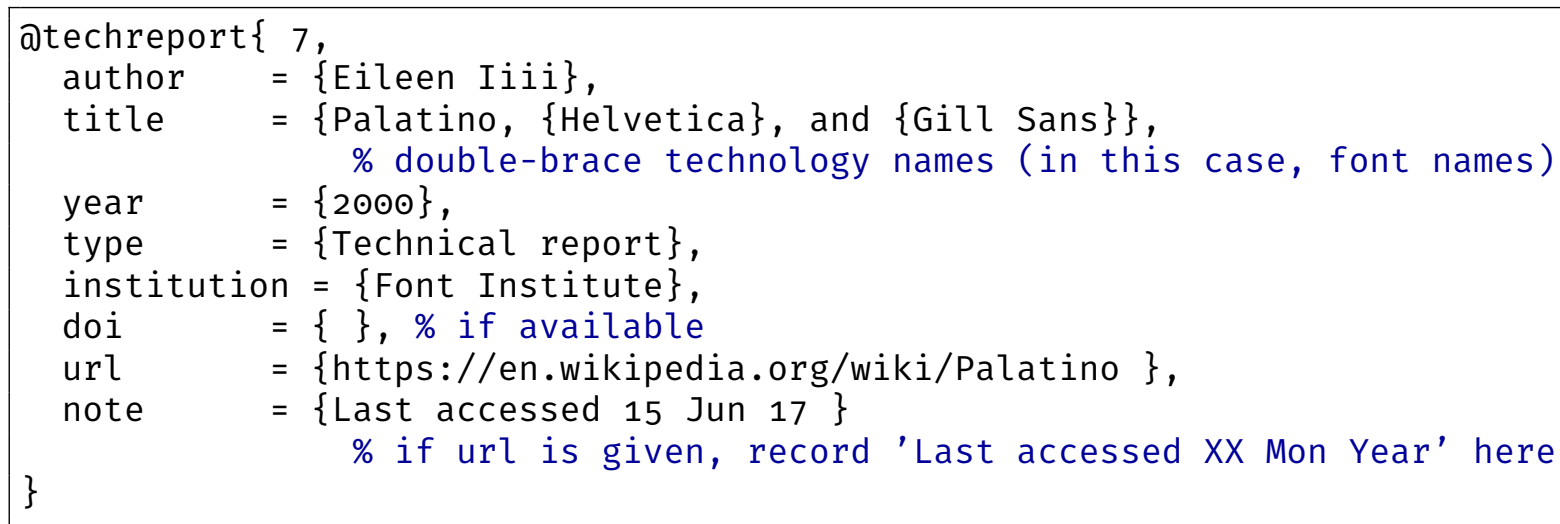

\subsection{Webpages and online resources}

South African Institute for Computer Scientists and Information Technologists (1998) is provided by the following BibTex code:

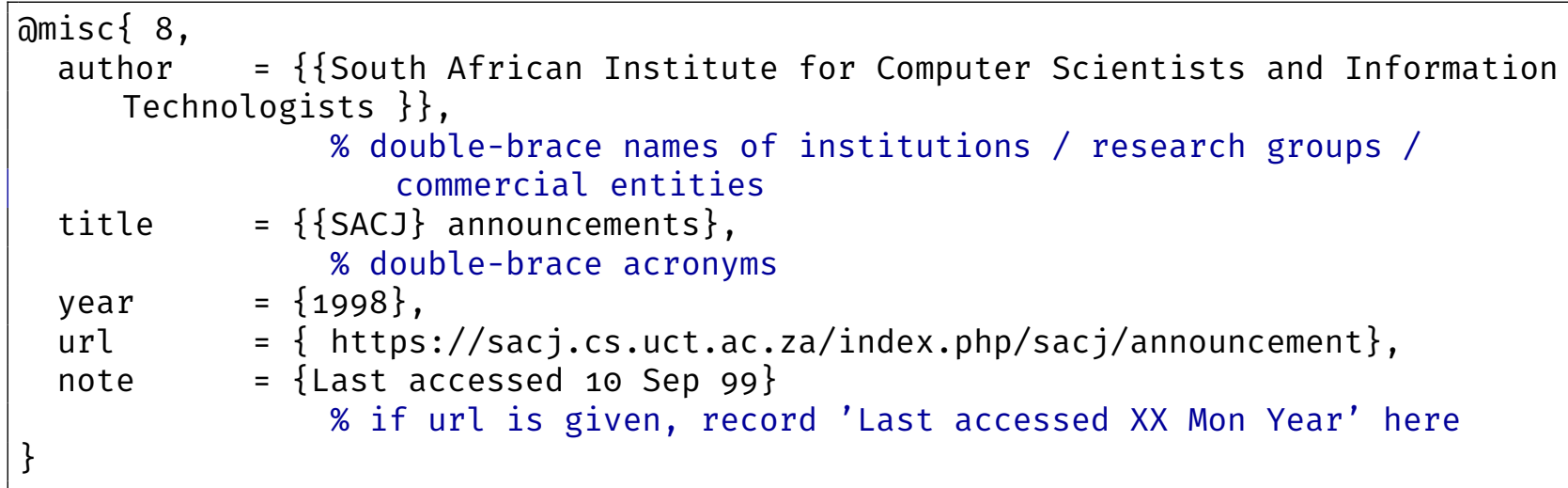

\section{References}

Aaaa, P. Q., \& Bbbb, R. S. (2047). The quick brown fox jumps over the lazy dog: An overview of keyboard test procedures in the short twentieth century. Historical Perspectives On Quality Assurance, 17(3), 210-211. https://doi.org/001.0001/001.1.0001

Cccc, T. (2017). Think like a computer, scientist: Debugging Prolog, In 2017 IET 4th International Conference on Logic Programming, Toronto, Canada, 22-24 May. IET. https://doi. org/002.0002/002.2.0002

Dddd, U. (2008). Modern developments in modelling development (3rd). Megadodo. https://doi. org/003.0003/003.3.0003 
Eeee, V. (1998). Dude, where's my UART? (R. Ffff, Ed.). In R. Ffff (Ed.), Mastering on-target debugging for wireless nanodevices. Zarniwoop-Verlag.

Gggg, X. (1888). Procedural power solutions: A multidisciplinary intervention (Master's thesis). Xyzzy Institute of Technology. Vancouver.

Hhhh, W. (1999). Quantitative studies in quantum quintessence (Doctoral dissertation). Qwerty College. Wellington.

Iiii, E. (2000). Palatino, Helvetica, and Gill Sans (Technical report) [Last accessed 15 Jun 17]. Font Institute. Last accessed 15 Jun 17. https://en.wikipedia.org/wiki/Palatino

South African Institute for Computer Scientists and Information Technologists. (1998). SACJ announcements [Last accessed 10 Sep 99]. https://sacj.cs.uct.ac.za/index.php/sacj/ announcement 